\title{
High luteinizing hormone levels are associated with Thyroid Nodules in Male Patients with Type 2 Diabetes
}

rendong zheng

Jiangsu Province Hospital on Integration of Chinese and Western Medicine, Nanjing University of Traditional Chinese Medicine

\section{Huifeng Zhang}

Jiangsu Province Academy of Traditional Chinese Medicine

lin cao

Jiangsu Province Academy of Traditional Chinese Medicine

wenbo ding

Jiangsu Province Academy of Traditional Chinese Medicine

yuguo wang

Jiangsu Province Academy of Traditional Chinese Medicine

Chao Liu ( $\nabla$ profliuchao@163.com )

\section{Research article}

Keywords: Diabetes, thyroid nodules, sex hormones, luteinizing hormone, insulin resistance

Posted Date: June 11th, 2020

DOI: https://doi.org/10.21203/rs.3.rs-32703/v1

License: (c) (1) This work is licensed under a Creative Commons Attribution 4.0 International License. Read Full License 


\section{Abstract}

Objective. Thyroid nodules are closely related to type 2 diabetes. However, the relationship between sex hormone levels and thyroid nodules has never been fully examined in males with type 2 diabetes. The purpose of the present study was to observe the association between sex hormone levels and thyroid nodules in male patients with type 2 diabetes.

Methods. A total of 490 patients were enrolled, A total of 228 patients (46.5\%) had thyroid nodules, of which $192(39.2 \%)$ had a solid benign nodule, $30(6.1 \%)$ had a cystic nodule, and $6(1.2 \%)$ had a cancerous nodule, 262 patients (53.5\%) were without thyroid nodules $₫ W$ We evaluated thyroid nodules using ultrasound, and blood glucose, blood lipids, serum insulin, sex hormones were measured in all patients. The correlations between the hormone levels and thyroid nodules were analyzed.

Results. Compared with the non-thyroid nodules group, fasting insulin (FINS), homeostasis model assessment of insulin resistance (HOMA-IR) and luteinizing hormone (LH) levels were significantly elevated in the thyroid nodules group. Pearson correlation analysis found the incidence of thyroid nodules was positively correlated with FINS, HOMA-IR and LH. High LH levels was associated with thyroid nodules after controlling for FINS and HOMA-IR.

Conclusion. These data suggest that high LH level plays an important role in the development of thyroid nodules. FINS, HOMA-IR and LH are independent risk factors for thyroid nodules in male patients with type 2 diabetes.

\section{Introduction}

In recent years, the incidence of thyroid nodules has gradually increased with improvements in imageology $(1,2)$. Numerous studies suggest a prevalence of $19-67 \%$ when ultrasound is used for diagnosis(3). Approximately $5-15 \%$ may be malignant lesions(4). In China, meta-analysis suggests that the prevalence of goiters was $22.8 \%$ before 1996 and $12.6 \%$ after and that the prevalence of thyroid nodules is $22.7 \%(5)$. In general, the prevalence of thyroid nodules is affected by race, age, gender, region, history of radiation exposure, iodine intake and other factor s(6-8) .

Recently, the relationship between thyroid nodules and diabetes has become a focus of attention. Studies have found that type 2 diabetes in particular is closely related to the development of thyroid nodules. The incidence of thyroid nodules is significantly higher in patients with diabetes than in patients without diabetes(9), and thyroid cancer prevalence is higher in patients with glucose metabolism disorders, which are considered a risk factor for thyroid cancer $(10,11)$.

It is generally known that the regulation of physiology and pathology is a dynamic and complicated system, drawing on the nervous, immune and endocrine systems. The endocrine hormones, especially the sex hormones, plays an important role in the progress of metabolic diseases. Compared to the general population, patients with diabetes are more likely to experience imbalances in several hormones, such as 
insulin, sex hormones, and so on $(12,13)$. Currently, it is unclear whether these hormones are associated with thyroid nodules in diabetes. Therefore, it is especially important to establish whether these sex hormones are independent risks factors for thyroid nodules in type 2 diabetes in order to explain the pathogenesis of thyroid nodules.

\section{Research Design And Methods}

\section{Subjects}

Four hundred and ninety male patients with type 2 diabetes were recruited between January 2015 and June 2019 from the Department of Endocrinology and Metabolism. Informed consent was obtained from all patients, and the study was approved by the Ethics Committee of the Jiangsu Province Hospital on Integration of Chinese and Western Medicine, Nanjing University of Traditional Chinese Medicine. All of the patients were assigned either to the thyroid nodules group (192 patients with solid benign nodule, 30 patients with cystic nodule, and 6 patients with cancerous nodule) or the non-thyroid nodules group (262 patients).

Any of the following conditions constituted an exclusion criterion: 1) Acute or chronic liver or kidney dysfunction. 2) Cardiac insufficiency. 3) Acute complications of diabetes. 4) History of thyroid, sex gland or adrenal disease. 5) Infectious and autoimmune diseases. 6) Patients with Hormone supplement or replacement.

\section{Hormonal and biochemical assays}

Patients' height, weight and blood pressure were measured. To calculate body mass index (BMI), we used the following formula: weight $(\mathrm{kg}) /$ height $(\mathrm{m})^{2}$.

To measure biochemical indicators, patients were tested in the morning (between 7:00 and 8:00 AM) after fasting 8-10 h. A range of biochemical indicators were measured, including fasting blood glucose ( FBG), glycosylated hemoglobin (HbA1c), liver and kidney function, cholesterol (TC), triglycerides (TG), lowdensity lipoprotein cholesterol (LDL-c), high density lipoprotein cholesterol (HDL-c). Blood glucose was determined by the glucose oxidase method, and blood lipid composition was determined by enzymatic analysis (Roche Diagnostics, Mannheim, Germany).

To measure fasting insulin (FINS, normal range: 2.6-24.9 $\mu / U / L)$, HOMA insulin resistance index (HOMAIR) was calculated using the formula fasting plasma glucose xfasting plasma insulin / 22.5 (14). Sex hormones were tested in the morning (between 7:00-8:00 AM) after fasting 8-10 h. We used chemiluminescence (Siemens Healthcare Diagnostics, New York, USA) to measure the following: total testosterone (TT, normal range: 8.64-29.0 $\mathrm{nmol} / \mathrm{L}$ ), sex hormone- binding globulin (SHBG, normal range: 14.5-48.4 $\mathrm{nmol} / \mathrm{L}$ ), progesterone (P, normal range: 0.7-4.3 $\mathrm{nmol} / \mathrm{L}$ ), prolactin (PRL, normal range: 86-324.0 $\mu / U / L)$, luteinizing hormone ( $\mathrm{LH}$, normal range: 1.7-8.6 $\mathrm{m} / \mathrm{U} / \mathrm{L})$, follicle-stimulating hormone ( $\mathrm{FSH}$, normal range: 1.5-12.4 $\mathrm{m} / \mathrm{U} / \mathrm{ml}$ ), and estradiol (E2, normal range: 28-156 $\mathrm{pmol} / \mathrm{L})$. 


\section{Ultrasonographic assessments}

Thyroid ultrasonography was performed on site by 2 fulltime ultrasound doctors at the Jiangsu Province Hospital on Integration of Chinese and Western Medicine, Nanjing University of Traditional Chinese Medicine. Thyroid nodules were evaluated for size, number, shape, margin and microcalcification using a HI VISION Preirus ultrasound machine (Japan) with 10-13 MHZ probes.

\section{Statistical analysis}

Data analysis was conducted using SPSS 22.0 , and p-values $₫ 0.05$ were considered statistically significant. All values are expressed as the mean \pm SD for the quantitative variables and as a percentage for the qualitative variables. The characteristics of the two groups were compared by t-tests or nonparametric Mann-Whitney tests for the quantitative variables and Fisher's exact tests or $\chi 2$ tests for the qualitative variables. One-way ANOVA was used for comparison across multiple groups. Factors associated with thyroid nodules were estimated using logistic regression.

\section{Results}

\section{Subject characteristics}

The current study included 490 diabetic patients. A total of 228 patients $(46.5 \%)$ had thyroid nodules, of which $192(39.2 \%)$ had a solid benign nodule, $30(6.1 \%)$ had a cystic nodule, and $6(1.2 \%)$ had a cancerous nodule. We excluded patients with cystic nodule and cancerous nodule in this study. Overall, 262 patients (53.5\%) were without thyroid nodules. In this study, we excluded cystic thyroid nodules and thyroid cancer, only evaluated the largest nodule in patients with multiple nodules. Patients in the thyroid nodules group did not differ from those in the non-thyroid nodules group in terms of age, duration of diabetes, BMI, FBG, P2hBG, HbAlc, TG, TC, HDL-c and LDL-c levels (Table 1).

\section{Comparison of insulin and HOMA-IR in the two groups}

A comparison of the FINS and HOMA-IR of patients with and without thyroid nodules is shown in Table 1. Compared with the non-thyroid nodules group, FINS and HOMA-IR levels were elevated in the thyroid nodules group ( $p \otimes 0.05$ ).

\section{Comparison of sex hormones in the two groups}

As shown in Table 2, compared with the non-thyroid nodules group\&LH levels were significantly higher in the thyroid nodules group ( $p \otimes 0.05$ ), while TT, SHBG, FSH, P, PRL, E2, DHEA levels were not significantly different $(p \otimes 0.05)$. 


\section{Comparison of FINS, HOMA-IR, and sex hormones in various thyroid nodules groups}

All patients were divided into four groups on the basis of the size of their thyroid nodule (G0: no thyroid nodules,G1: thyroid nodules present but $\otimes 1.0 \mathrm{~cm} \otimes \mathrm{G} 2$ : thyroid nodules between 1.0 and $2.0 \mathrm{~cm} \otimes \mathrm{G} 3$ : thyroid nodules $\triangle 2.0 \mathrm{~cm}$ ). Compared with the G0, FINS, HOMA-IR and LH levels were higher in the G1, G2 and G3; Compared with the G1, FINS, HOMA-IR and LH levels were higher in the G3 ( $p \bowtie 0.05$, Figure 1)『while FSH, TT and E2 levels did not differ (Figure 1) .

\section{Comparison of the incidence of thyroid nodules across levels of FINS, HOMA-IR, LH and TT}

All cases were divided into four quartile groups according to FINS, HOMA-IR, LH and TT (Q1 group = 025th; Q2 group = 26-50th; Q3 group = 51-75th; Q4 group = 76-100th percentile). Compared with the Q1 group, the incidence of thyroid nodules was higher in the Q4 group of FINS, HOMA-IR and LH $(p<0.05)$ (Figure 2, A-C). but the incidence of thyroid nodules did not differ in the Q4 group of TT ( $p \otimes 0.05$ ) (Figure $2, \mathrm{D})$.

\section{Correlation and regression analysis}

Pearson correlations showed that the size of thyroid nodules was positively correlated with FINS, HOMAIR, LH levels ( $r=0.215, p=0.003 \rrbracket r=0.387 \rrbracket p=0.001, r=0.181, p=0.012$; respectively ) ( Figure 3 ) , while size of thyroid nodules was not correlated with TT ( Figure 3), FSH, SHBG, or E2 ( $r=0.133, p=0.066 ; r=0.033 \rrbracket$ $p=0.650 ; r=0.092 \rrbracket p=0.203 \rrbracket r=0.050, p=0.495$, respectively).

We conducted a logistic regression treating the presence of a thyroid nodule as the dependent variable, and age, duration of diabetes, BMI, FBG, HbA1c, FINS, HOMA-IR, TG, LDL-c, HDL-c, TT, LH, FSH and E2 as the independent variables. This analysis showed that FINS, HOMA-IR, LH were independent risk factors for thyroid nodules, and LH were associated with thyroid nodules after controlling for FINS, HOMA-IR (Table 3).

\section{Discussion}

With the widespread use of ultrasonography machines in clinical practice, thyroid nodules are being discovered with increasing frequency(15). Studies have shown that many risk factors may affect the development of nodules in women $(16,17)$. The most current studies focus on thyroid nodules in women. However, the relationship between type 2 diabetes in men and thyroid nodules has rarely been examined. In this study, we investigated the prevalence of thyroid nodules in male patients with type 2 diabetes, finding a prevalence of $45 \%$ in this sample, which is significantly higher than in the general population(18) . 
BMI may be involved in the pathogenesis of thyroid nodules. However, our results showed that thyroid nodules were not correlated with BMI after adjusting for age. Moreover, we did not find a relationship between nodules and hypertension or dyslipidemia, perhaps due to our smaller sample size. This is not to deny that insulin resistance is an independent risk factor for thyroid nodules in patients with obesity(19).

Our results showed that serum insulin levels and HOMA-IR were associated with thyroid nodules. It is well established that insulin resistance is a major risk factor for thyroid nodules, although some have argued against the claim that insulin resistance and hyperglycemia can result in thyroid nodules. Arduc $A$ et al. reported that HOMA-IR was similar in patients with and without thyroid nodules(20). However, Semra has argued for an association, noting that thyroid volume is enlarged in patients with metabolic syndrome and identifying insulin resistance as an independent risk factor for thyroid nodules(21). Our results showed that FINS is positively correlated with the size of thyroid nodules. Our previous work has shown that IR and hyperglycemia were positively correlated with the flow patterns, resistive index and vascular index of thyroid nodules in a cross-sectional, population-based study(22). Additionally, Meterformin produces a significant decrease in the nodule size in patients with IR and small thyroid nodules. Moreover, Meterformin alone or combined with levothyroxine (L-T4) can improve insulin resistance and reduce thyroid nodules, and Meterformin decreases insulin-induced cell proliferation of thyroid cancer cells and cancer stem cells $(23,24)$.

Insulin, as a growth factor, can stimulate growth of various cells, including thyroid cells. Experiments show that thyroid cells incubated with TSH alone proliferate slowly, while cells incubated with TSH plus insulin increase significantly(25). On the other hand, insulin resistance, with increased levels of insulin and insulin-like growth factor, may increase the risk of thyroid cancer(26). Therefore, insulin resistance may be among the factors (including proinflammatory factor, fat cell factors and thyroid-stimulating hormone) that play a key role in the development of thyroid cancer $(22,27)$.

In this study, the relationship between sex hormones and thyroid nodules in type 2 diabetes was investigated. Scholars think the high prevalence of thyroid nodules in women during pregnancy, lactation and menstrual periods may be caused by periodic increases in thyroid hormones and other endocrine changes, but the relationship between estrogen levels and thyroid nodules is not yet clear(28). Estrogen might be an important element in the pathogenesis of thyroid nodules. Estrogen can promote thyroid cell mitosis through the mitogen-activated protein kinase pathway in benign and malignant nodules $(29,30)$. This may be the reason that the incidence of thyroid nodules is higher in women than in men. However, the evidence to date indicates that there isn't a relationship between thyroid nodules and estrogen in male patients.

Significantly, LH levels were higher in the thyroid nodules group. Compared with the low-LH group, the incidence of thyroid nodules increased significantly in the high-LH group. To the best of our knowledge, based on the few reports on sex hormones and male thyroid nodules, this paper is the first to study the relationship between LH and thyroid nodules in males with type 2 diabetes. Previous studies showed that thyroid adenoma is often accompanied by elevated levels of serum FSH and $\mathrm{LH}$ and the expression of 
FSHR and LHR in female patients (31). It has been reported that hormones such as LH, FSH, and TSH can promote the proliferation of various tumor cells, including lung cancer, thyroid cancer, cervical cancer, breast cancer, and prostate cancer(32). One experiment found that $50 \mathrm{miu} / \mathrm{ml}$ of LH significantly regulates VEGF and slit2 expression in epithelial ovarian cancer cell lines, activating the PI3K/AKT-mTOR signaling pathway and promoting tumor growth(33). Recently, Oguz et al. investigated the effect of hCG treatment or testosterone treatment on thyroid volume in males with isolated hypogonadotropic hypogonadism. Their results show thyroid volume was higher in hCG-treated patients than in testosterone-treated patients, but thyroid volume did not differ before and after testosterone treatment (34) . Further research is needed to elucidate the biological mechanisms underlying the associations between LH and FSH and thyroid nodules in men.

There are some limitations to our study. First, this was a retrospective study, we did not have the data of the general population, and lack of comparison between the diabetes and the normal control subjects; Second, we know iodine level is closely associated with thyroid nodules, we did not have access to such information of iodine intake in the patients although Jiangsu is a relatively iodine sufficient area; At last, we did not talk about the relationship between thyroid nodules and income, education, smoking and alcohol drinking.

\section{Conclusions}

In the present study, we conclude that there is a close relationship between thyroid nodules and type 2 diabetes. Our research indicates that hormonal imbalance plays an important role in the development of thyroid nodules. Our results showed that FINS, HOMA-IR, LH, but not FSH, TT, E2, are independent risk factors for thyroid nodules in male patients with type 2 diabetes.

\section{Declarations}

\section{Conflict of Interests}

The authors declare that they have no competing interests.

\section{Data Availability statement}

The data used to support the findings of this study are available from the corresponding author upon request.

\section{References}

1. Russ G, Leboulleux S, Leenhardt L, Hegedüs L.Thyroid incidentalomas: epidemiology, risk stratification with ultrasound and workup. Eur Thyroid J 3:154-63,2014.

2. Cohen RN, Davis AM. Management of Adult Patients With Thyroid Nodules and Differentiated Thyroid Cancer. JAMA 317:434-35,2017. 
3. Burman KD, Wartofsky L.CLINICAL PRACTICE. Thyroid Nodules. N Engl J Med 373:2347-56, 2015.

4. Moubayed SP, Urken ML.Thyroid nodules. CMAJ 88:1259,2016.

5. Gandolfi PP, Frisina A, Raffa M, Renda F, Rocchetti O, Ruggeri C, et al. The incidence of thyroid carcinoma in multinodular goiter: retrospective analysis. Acta Biomed 75:114-7,2004.

6. Utiger RD. The multiplicity of thyroid nodules and carcinomas. N Engl J Med 352:2376-8,2005.

7. Niedziela M. Thyroid nodules. Best Pract Res Clin Endocrinol Metab 28:245-77, 2014.

8. Pemayun TG. Current Diagnosis and Management of Thyroid Nodules. Acta Med Indones 48:247-57, 2016.

9. Junik R, Kozinski M, Debska-Kozinska K. Thyroid ultrasound in diabetic patients without overt thyroid disease. Acta Radiol 47:687-91, 2006.

10. Duran A01, Anil C, Gursoy A, Nar A, Altundag O, Tutuncu NB. The relationship between glucose metabolism disorders and malignant thyroid disease. Int J Clin Oncol18:585-9,2013.

11. Blanc E, Ponce C, Brodschi D, Nepote A, Barreto A, Schnitman M, et al. Association between worse metabolic control and increased thyroid volume and nodular disease in elderly adults with metabolic syndrome. Metab Syndr Relat Disord13:221-6, 2015.

12. Lee ZS, Chan JC, Yeung VT, Chow CC, Lau MS, Ko GT, et al. Plasma insulin, growth hormone, cortisol, and central obesity among young Chinese type 2 diabetic patients. Diabetes Care 22:1450-7,1999.

13. Khatiwada S, Kc R, Sah SK, Khan SA, Chaudhari RK, Baral N, et al.Thyroid Dysfunction and Associated Risk Factors among Nepalese Diabetes Mellitus Patients. Int J Endocrinol2015:570198,2015.

14. Wallace TM, Levy JC, Matthews DR. Use and abuse of HOMA modeling. Diabetes Care27(6):1487$95,2004$.

15. Franco Uliaque C, Pardo Berdún FJ, Laborda Herrero R, Lórenz CP. Usefulness of ultrasonography is the evaluation of thyroid nodules. Radiologia58:380-388, 2016.

16. Carlé A, Krejbjerg A, Laurberg P. Epidemiology of nodular goitre. Influence of iodine intake. Best Pract Res Clin Endocrinol Metab28: 465-79, 2014.

17. Botrugno I, Lovisetto F, Cobianchi L, Zonta S, Klersy C, Vailati A, et al. Incidental carcinoma in multinodular goiter: risk factors. Am Surg 77:1553-8, 2011.

18. Guo H, Sun M, He W, Chen H, Li W, Tang J, Tang W, et al. The prevalence of thyroid nodules and its relationship with metabolic parameters in a Chinese community-based population aged over 40 years. Endocrine45:230-5, 2014.

19. Ayturk S, Gursoy A, Kut A, Anil C, Nar A, Tutuncu NB. Metabolic syndrome and its components are associated with increased thyroid volume and nodule prevalence in a mild-to-moderate iodinedeficient area. Eur J Endocrinol 161:599-605,2009.

20. Arduc A, Isik S, Ozuguz U, Tutuncu YA, Kucukler FK, Ozcan HN, et al. Relationship between thyroid nodules and non-functioning adrenal incidentalomas and their association with insulin resistance. Endocr Res39:99-104, 2014. 
21. Anil C, Akkurt A, Ayturk S, Kut A, Gursoy A. Relationship between thyroid nodules and non-functioning adrenal incidentalomas and their association with insulin resistance. Endocr Res 62:970-5, 2014.

22. Wang K, Yang Y, Wu Y, Chen J, Zhang D, Mao X, et al.The association between insulin resistance and vascularization of thyroid nodules. J Clin Endocrinol Metab 100:184-92, 2015.

23. Rezzónico J, Rezzónico M, Pusiol E, Pitoia F, Niepomniszcze H. Metformin treatment for small benign thyroid nodules in patients with insulin resistance.Metab Syndr Relat Disord 9:69-75,2011.

24. Chen G, Xu S, Renko K, Derwahl M. Metformin inhibits growth of thyroid carcinoma cells, suppresses self-renewal of derived cancer stem cells, and potentiates the effect of chemotherapeutic agents. J Clin Endocrinol Metab 9 7:E510-20, 2012.

25. Yasar HY, Ertuğrul O, Ertuğrul B, Ertuğrul D, Sahin M. Insulin resistance in nodular thyroid disease. Endocr Res36:167-74, 2011.

26. Pazaitou-Panayiotou K, Polyzos SA, Mantzoros CS. Mantzoros. Obesity and thyroid cancer: epidemiologic associations and underlying mechanisms. Obes Rev14 :1006-22, 2013.

27. Pappa, M. Alevizaki. Obesity and thyroid cancer: a clinical update.Thyroid 24: 190-99,2014.

28. Rajoria S, Suriano R, Parmar PS, Wilson YL, Megwalu U, Moscatello A,et al. 3,3'-diindolylmethane modulates estrogen metabolism in patients with thyroid proliferative disease: a pilot study. Thyroid21:299-304,2011.

29. Rajoria S, Suriano R, George A, Shanmugam A, Schantz SP, Geliebter J, et al. Estrogen induced metastatic modulators MMP-2 and MMP-9 are targets of 3,3'-diindolylmethane in thyroid cancer. PLoS One6:e15879, 2011.

30. Derwahl M, Nicula D. Estrogen and its role in thyroid cancer. Endocr Relat Cancer 21: T273-83, 2014.

31. Liu J, Chen G, Meng XY, Liu ZH, Dong S. Serum levels of sex hormones and expression of their receptors in thyroid tissue in female patients with various types of thyroid neoplasms.Pathol Res Pract 210:830-5, 2014.

32. Govindaraj V, Arya SV, Rao AJ. Differential action of glycoprotein hormones: significance in cancer progression. Horm Cancer 5:1-10, 2014.

33. Liao H, Zhou Q, Gu Y, Duan T, Feng Y. Luteinizing hormone facilitates angiogenesis in ovarian epithelial tumor cells and metformin inhibits the effect through the mTOR signaling pathway. Oncol Rep 27:1873-8, 2012.

34. Oguz A, Tuzun D, Sahin M, Bulbul N, Celik A, Guvenc N,et al. Should human chorionic gonadotropine treatment increase thyroid volume? Arch Endocrinol Metab59:482-6, 2015.

\section{Tables}


Table 1. Comparison of indicators of glucolipid and insulin in two groups

\begin{tabular}{llll}
\hline & Thyroid nodules & Non-thyroid nodules & $p$ \\
\hline$N$ & 192 & 262 & 0.902 \\
HbA1c (\%) & $9.16 \pm 2.35$ & $9.74 \pm 2.54$ & 0.881 \\
\hline$F B G(\mathrm{mmol} / \mathrm{L})$ & $8.27 \pm 234$ & $8.46 \pm 2.39$ & 0.696 \\
\hline$P B G(\mathrm{mmol} / \mathrm{L})$ & $16.15 \pm 4.03$ & $16.42 \pm 4.13$ & 0.854 \\
\hline$T C \square \mathrm{mmol} / \mathrm{L} \square$ & $4.57 \pm 1.25$ & $4.37 \pm 1.03$ & 0.062 \\
\hline$T G \square \mathrm{mmol} / \mathrm{L} \square$ & $2.38 \pm 2.92$ & $2.14 \pm 1.76$ & 0.091 \\
\hline$L D L-c \square \mathrm{mmol} / \mathrm{L} \square$ & $2.61 \pm 0.62$ & $2.52 \pm 0.75$ & 0.134 \\
\hline$H D L-C \square \mathrm{mmol} / \mathrm{L} \square$ & $1.06 \pm 0.39$ & $1.14 \pm 0.35$ & 0.092 \\
\hline FINS $(\mu I \mathrm{U} / \mathrm{L})$ & $9.98 \pm 6.06$ & $7.23 \pm 7.32$ & 0.045 \\
\hline$H O M A-I R$ & $3.82 \pm 2.23$ & $3.01 \pm 2.08$ & 0.039 \\
\hline
\end{tabular}

Data are presented as the mean \pm SD in each group. HbA1c, glycosylated hemoglobin; FBG, fasting glucose; PBG, postprandial blood glucose; FINS, fasting insulin; PINS, postprandial insulin; TC, cholesterol; TG, triglyceride; LDL-c, low-density lipoprotein cholesterol; HDL-C, high-density lipoprotein cholesterol; HOMA-IR, HOMA insulin resistance index.

Table 2. Comparison of sex hormone, thyroid hormone and adrenal hormone in two groups

\begin{tabular}{llll}
\hline & Thyroid nodules & Non-thyroid nodules & $p$ \\
\hline$N$ & 192 & 262 & \\
$T T(\mathrm{nmol} / \mathrm{L})$ & $19.39 \pm 1.99$ & $18.01 \pm 5.14$ & 0.106 \\
\hline$S H B G(\mathrm{nmol} / \mathrm{L})$ & $28.89 \pm 12.54$ & $24.92 \pm 23.63$ & 0.118 \\
\hline$P(\mathrm{nmol} / \mathrm{L})$ & $1.46 \pm 0.65$ & $1.27 \pm 0.56$ & 0.079 \\
\hline$P R L(\mu / \mathrm{U} / \mathrm{L})$ & $301.34 \pm 98.79$ & $292.02 \pm 122.95$ & 0.613 \\
\hline$L H(\mathrm{~m} / \mathrm{U} / \mathrm{L})$ & $7.96 \pm 3.31$ & $6.15 \pm 3.41$ & 0.045 \\
\hline$F S H(\mathrm{~m} / \mathrm{U} / \mathrm{L})$ & $6.64 \pm 5.29$ & $5.99 \pm 3.90$ & 0.062 \\
\hline$E 2(\mathrm{pmol} / \mathrm{L})$ & $112.51 \pm 37.71$ & $116.16 \pm 49.16$ & 0.141 \\
\hline$D H E A(\mu \mathrm{mol} / \mathrm{L})$ & $6.06 \pm 3.06$ & $5.32 \pm 2.81$ & 0.170 \\
\hline
\end{tabular}

Data are presented as the mean $\pm S D$ in each group. TT, total testosterone; SHBG, sex hormone binding globulin; P, progesterone; PRL, prolactin; LH, luteinizing hormone; FSH, follicle-stimulating hormone; E2, estradiol; FINS, fasting insulin; HOMA-IR, HOMA insulin resistance index. 
Table 3. Logistic regression analysis for thyroid nodules risk factor

\begin{tabular}{lllllll}
\hline & $\beta$ value & $S E$ & Wald & $p$ & OR & $95 \% \mathrm{Cl}$ \\
\hline FINS & 0.264 & 0.365 & 5.129 & 0.027 & 1.352 & $1.164-1.295$ \\
\hline HOMA-IR & 0.139 & 0.053 & 5.625 & 0.012 & 1.426 & $1.053-1.352$ \\
\hline LH & 0.162 & 0.037 & 6.146 & 0.043 & 1.275 & $1.038-1.217$ \\
\hline TT & 0.215 & 0.081 & 7.213 & 0.061 & 1.068 & $0.132-1.264$ \\
\hline
\end{tabular}

Note: FINS, fasting insulin; HOMA-IR, HOMA insulin resistance index; LH, luteinizing hormone; TT, total testosterone.

\section{Figures}
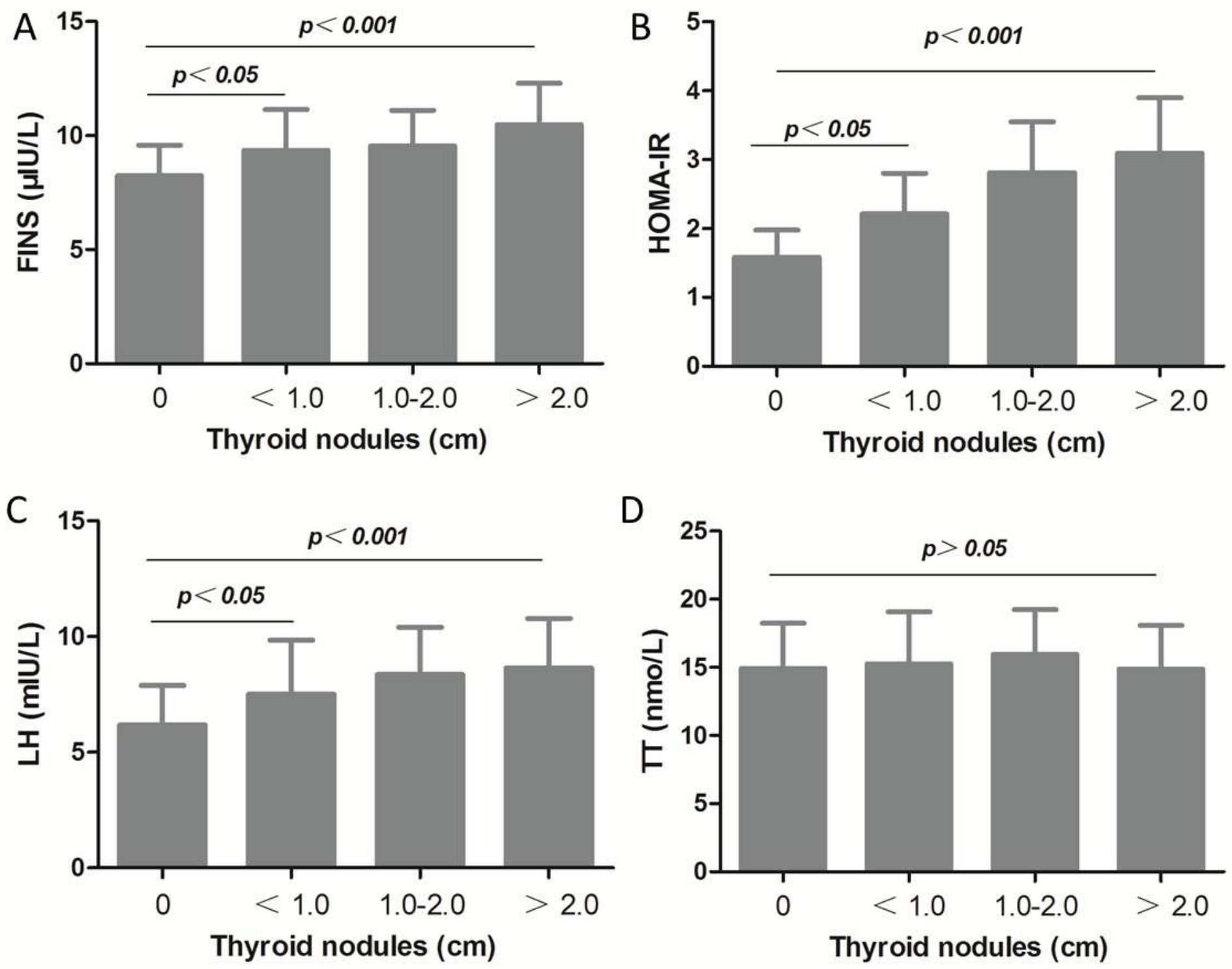

Figure 1 
Compared with the G1, FINS, HOMA-IR and LH levels were higher in the G3 ( $p \otimes 0.05)$, while FSH, TT and E2 levels did not differ.
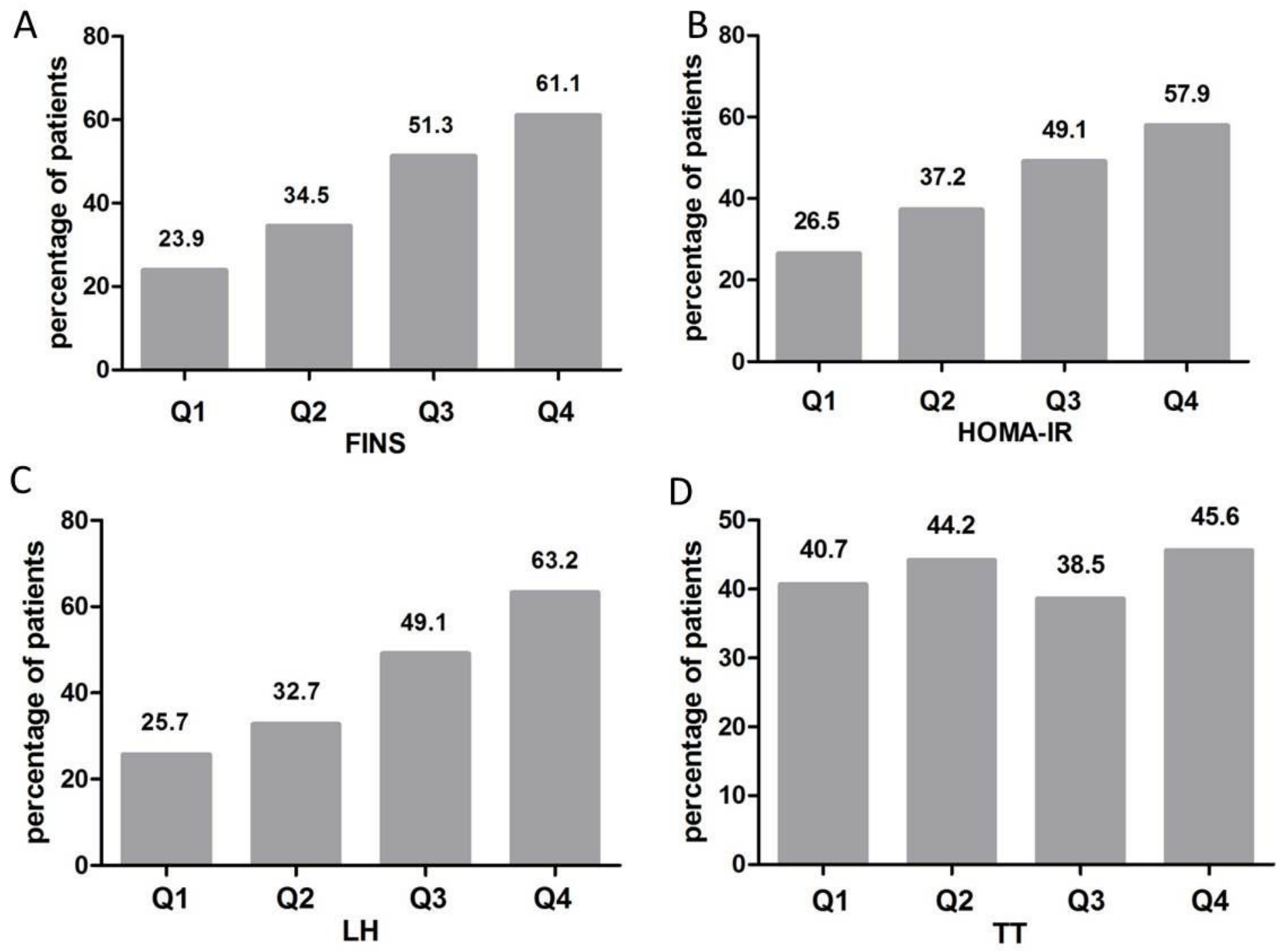

Figure 2

Compared with the Q1 group, the incidence of thyroid nodules was higher in the Q4 group of FINS, HOMAIR and LH $(p<0.05)$ (Figure 2, A-C). but the incidence of thyroid nodules did not differ in the Q4 group of TT $(p \otimes 0.05)(D)$. 

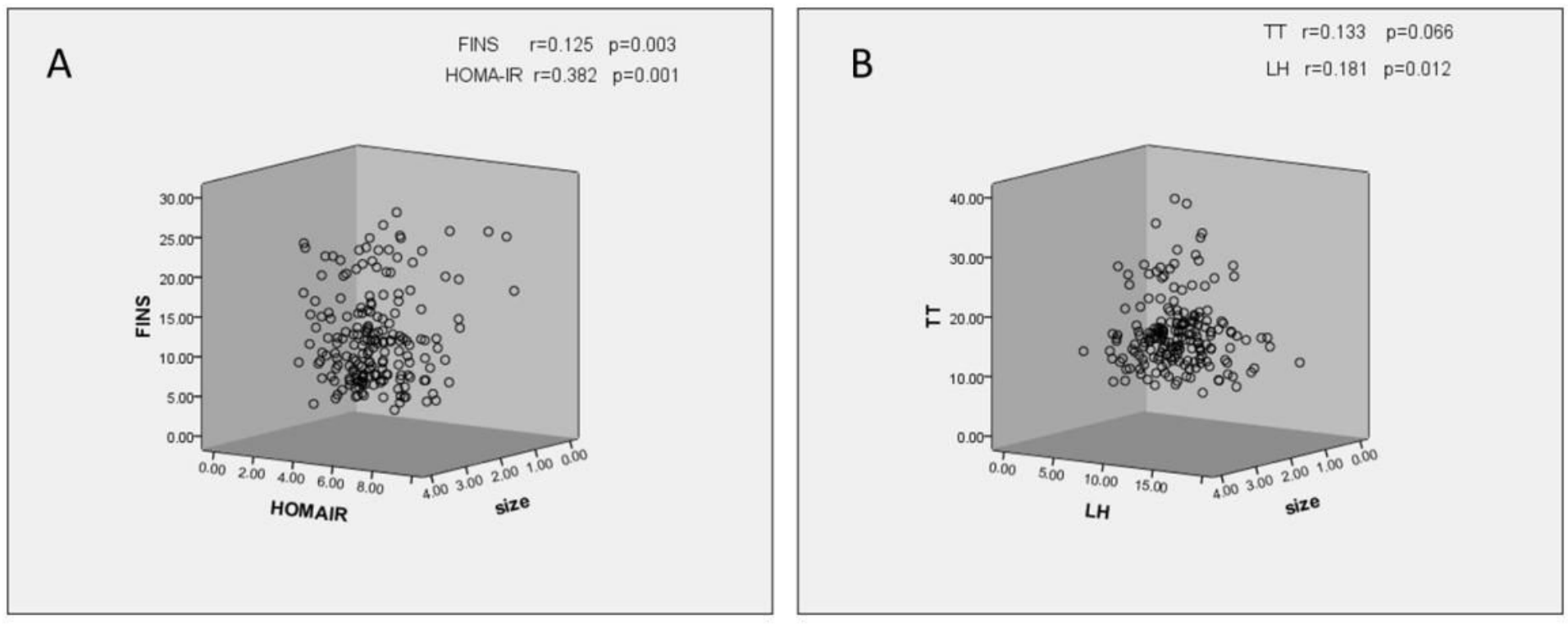

Figure 3

Pearson correlations showed that the size of thyroid nodules was positively correlated with FINS, HOMAIR, LH levels ( $r=0.215, p=0.003 \bigotimes r=0.387 \rrbracket p=0.001, r=0.181, p=0.012$; respectively $)$, while size of thyroid nodules was not correlated with TT, FSH, SHBG, or E2 ( $r=0.133, p=0.066 ; r=0.033 \rrbracket p=0.650 ; r=0.092 \rrbracket$ $p=0.203 \otimes r=0.050, p=0.495$, respectively). 